REFERENCIA: Sebastiá-Alcaraz, R. \& García-Rubio, J. (2021). La relevancia de la coordinación docente en el Máster de Formación del Profesorado de Secundaria en Geografía e Historia. ENSAYOS, Revista de la Facultad de Educación de Albacete, 36(2), 113-129. Enlace web: http://www.revista.uclm.es/index.php/ensayos - Consultada en fecha (dd-mm-aaaa)

\title{
LA RELEVANCIA DE LA COORDINACIÓN DOCENTE EN EL MÁSTER DE FORMACIÓN DEL PROFESORADO DE SECUNDARIA EN GEOGRAFÍA E HISTORIA
}

\section{THE RELEVANCE OF TEACHING COORDINATION IN THE MASTER IN SECONDARY EDUCATION TEACHERS IN GEOGRAPHY AND HISTORY}

\author{
Rafael Sebastiá-Alcaraz \\ rafael.sebastia@ua.es \\ Facultad de Educación. Universidad de Alicante \\ Juan García-Rubio \\ Juan.Garcia-Rubio@uv.es \\ Facultad de Filosofía y Ciencias de la Educación. Universidad de Valencia
}

Recibido: 26/09/2021

Aceptado: 10/12/2021

\begin{abstract}
:
La coordinación entre el profesorado resulta fundamental para conseguir una formación idónea para el alumnado. Esta investigación se centra en el estudio de la coordinación docente en el Máster que habilita al estudiante para ejercer la docencia en la etapa de Educación Secundaria, y más en concreto en la especialidad de Geografía e Historia. A través de una metodología cuantitativa, pero utilizando también técnicas de análisis cualitativo, en la que la percepción del alumnado de dos universidades españolas resulta esencial, se han obtenido unos resultados que evidencian la falta de coordinación docente, provocando una repetición en los contenidos impartidos en las diferentes materias, al mismo tiempo que se produce la ausencia de otros que los estudiantes consideran fundamentales. La mejora de la formación inicial del futuro profesorado de Educación Secundaria requiere de un nuevo modelo de coordinación docente que sea capaz de armonizar las distintas materias impartidas en el Máster.
\end{abstract}

Palabras clave: formación inicial del profesorado; Máster del Profesorado; coordinación; Educación Secundaria; Ciencias Sociales.

\begin{abstract}
:
Coordination between teachers is essential to achieve suitable training for students. This research focuses on the study of teaching coordination in the Master that enables the student to teach in the Secondary Education stage, and more specifically in the specialty of Geography and History. Through a quantitative methodology, but also using qualitative analysis techniques, in which the perception of the students of two Spanish universities is essential, results have been obtained that show the lack of teaching coordination, causing a repetition in the contents taught in the different subjects as well as the absence of others that the students consider fundamental. Improving the initial training of future Secondary Education teachers requires a new model of teaching coordination that is capable of harmonizing the different subjects taught in the Master.
\end{abstract}

Keywords: initial teacher training; Master of Teaching; coordination; Secondary Education; Social Sciences. 


\section{Introducción}

La coordinación docente es un elemento primordial para conseguir la mejor formación del alumnado en todas las etapas educativas. La acción conjunta y armonizada del profesorado en el ámbito universitario es tan importante para la adquisición de unos aprendizajes adecuados y relevantes para los estudiantes como lo puede ser en otros periodos anteriores (Paricio Royo, 2012). El profesorado de las diferentes universidades, aunque de una forma tímida, cada vez es más proclive a abandonar el tradicional individualismo y a trabajar de forma más cooperativa en una mejor coordinación (Fuentes-Guerra, García, Llorent y Olivares, 2012; Lavié Martínez, 2004).

Uno de los aspectos esenciales de la coordinación entre docentes es el establecimiento de un currículum armonizado que atienda a las competencias requeridas del título de Grado o Máster. La consideración del currículum en la etapa universitaria como una sucesión de elementos estancos, sin ninguna conexión entre las diversas asignaturas, resulta enormemente perjudicial para el aprendizaje del alumnado (Jarauta Borrasca y Medina Moya, 2012). En este sentido, ya Bernstein (1973) diferenciaba dos tipos básicos de currículum: collection que podríamos traducir por currículum organizado en elementos completamente independientes y el otro tipo sería el denominado integrado, en el que los contenidos se entrelazaban relacionándose unos con otros.

Normalmente el conocimiento se fragmenta de forma artificial y los jóvenes estudiantes universitarios reciben un currículum collection o mosaico, donde las materias no tienen lazos en común, salvo que aparezcan de una manera casual. Sin embargo, en el currículum integrado el profesorado realiza el esfuerzo de coordinarse para conectar conocimientos de diferentes disciplinas con la misión de alcanzar aprendizajes más significativos para el alumno. Se podría pensar que la implantación de este currículum integrado podría hacer desaparecer las distintas materias, pero como señala Beane $(2005$, p. 63), "reivindicar el final del sistema de asignaturas separadas en la organización del currículum escolar no significa en absoluto rechazar ni abandonar las disciplinas del conocimiento".

No se trataría únicamente de consensuar contenidos y descartar sus posibles repeticiones entre las distintas materias, sino también de dialogar acerca de las actividades más convenientes algunas de las cuales se podrían realizar de forma articulada entre asignaturas-, así como de los tiempos en que se distribuyen durante el curso universitario, de manera que se evitara su concentración. La metodología a utilizar en el aula y la evaluación de las asignaturas son otras de las cuestiones sobre la que debatir y planificar en aras de ofrecer al alumnado un aprendizaje relevante. Esta coordinación docente implicaría "realizar un intercambio de conocimientos y un aprendizaje mutuo sobre el marco disciplinar en el que se ubican las distintas asignaturas" (Bolarín Martínez, Moreno Yus y Porto Currás, 2013, p. 457). Y por supuesto, la universidad debería apoyar en todo momento al profesorado desarrollando actividades para su formación y fomentando el intercambio de experiencias positivas ya implementadas (García Cid, 2013). Si realmente se apuesta desde la institución por una coordinación efectiva se tienen que poner a disposición del profesorado todos los medios y recursos para la consecución de este objetivo. La universidad debería también reconsiderar el que la implicación de al menos los responsables de la labor de coordinación estuviese reconocida bien en términos económicos o de carrera profesional, y no ser una responsabilidad más a añadir a la multitud de funciones del profesorado universitario (Torrego Ejido y Ruiz Esteban, 2011).

La coordinación puede ser oficialmente establecida -coordinación formal- o puede partir del propio profesorado -coordinación informal-. Esta última podría ser incluso más positiva que la anterior al no representar en ningún caso una mera formalidad para cumplir con lo establecido normativamente, sin que tuviese ningún efecto en la realidad del aula (Bolarín Martínez y 
Moreno Yus, 2015). A este respecto, Fuentes-Guerra et al. (2012) señalan que "estas situaciones colaborativas ficticias y artificiales tienen poca influencia en la mejora y el desarrollo profesional” (p. 397). No obstante, si se opta por una coordinación formal, también resulta de vital importancia la persona responsable de la coordinación y su compromiso, convicción y determinación en esta labor, pues se pueden establecer modos de actuación totalmente distintos dependiendo del acierto en su elección.

Torrego Ejido y Ruiz Esteban (2011, p. 33) señalan que "la imprescindible organización de los estudios universitarios en conjuntos de enseñanzas conectadas entre sí, conducentes en su conjunto a la adquisición de unas competencias (genéricas y específicas), exige la coordinación de la docencia a varios niveles". En relación con esta cuestión planteada, existe una coordinación vertical y otra horizontal. La coordinación vertical es la que se constituye entre los distintos cursos y materias de una titulación, mientras que la coordinación horizontal se establece entre un mismo curso y grupo (Bolarín et al., 2013; García Martín, 2015; López Esteban, 2016). Esta última coordinación resulta primordial, dado que implica trabajar de forma colaborativa entre profesorado que incluso pertenece a diferentes departamentos, pero que imparte una materia en el mismo nivel académico (García-Martínez, 2019). Se necesitan reuniones entre el profesorado de un mismo curso, que no sean únicamente debidas al compromiso institucionalizado, sino con la intención de fomentar la mejora efectiva del aprendizaje del alumnado.

El contexto de la investigación sobre la coordinación docente universitaria se ha circunscrito al Máster de Formación del Profesorado de Secundaria, que tras once años de su puesta en marcha en el curso 2009-10 por medio del Real Decreto 1393/2007 y la Orden ECI/3858/2007 necesita de evaluaciones tanto internas como externas y de establecer posibles mejoras, especialmente en los contenidos ofrecidos, en las metodologías empleadas e incluso en su tiempo de duración y acceso (Escudero Muñoz, Campillo Díaz y Sáez Carreras, 2019; Imbernón-Muñoz, 2019; González Sala, Bisquert, Haba-Osca y Osca-Lluch, 2020). En esta línea de posibles cambios a introducir en el Máster, Imbernón-Muñoz (2021) señala la necesidad de una formación inicial mucho más pegada a los contextos reales con los que se encontrará el futuro profesorado en las aulas de los centros educativos. Si la coordinación es primordial en cualquier titulación universitaria, todavía lo es más en este Máster de un único año y que conduce a la habilitación para ejercer la profesión docente en una etapa llena de retos y dificultades como es la Secundaria (Ávalos, Cavada, Pardo y Sotomayor, 2010; Perrenoud, 2012; Sarramona, 2007; Vaillant y Marcelo, 2015). En él se establecen diferentes especialidades dependiendo del Grado cursado previamente por el alumnado y de la tipología de las materias que quieren impartir más adelante como docentes de un centro de Enseñanza Secundaria (Sánchez Tarazaga, 2019).

La formación docente en el área de Ciencias Sociales, que es donde se ha ubicado la investigación, ha sido fruto más de la correlación de fuerzas de los departamentos que de la búsqueda de un proyecto coherente de currículum, como también ha ocurrido en el resto de las especialidades. Como señala Souto González (2017), la muy diferente estructura de los Másteres en España revela la carencia de un proyecto común que defina al profesorado de Ciencias Sociales, Geografía e Historia en la etapa de Secundaria. La organización departamental dificulta también la existencia de un corpus común, o contenidos estructurantes de referencia, para los diferentes saberes que integran las Ciencias Sociales. En la presente investigación hemos dirigido la mirada hacia el alumnado del Máster de Secundaria para conocer su visión privilegiada acerca de la coordinación que se ha establecido entre el profesorado de las distintas materias, y más en concreto en la especialidad de Geografía e Historia. 


\section{Método}

\subsection{Procedimiento}

El objetivo del estudio ha sido el análisis de la coordinación docente dentro del Máster de Profesorado de Secundaria en la especialidad de Geografía e Historia. Concretamente se han propuesto dos objetivos específicos: averiguar entre qué materias en la realización de esta coordinación existen más problemas, pudiendo conllevar la reiteración y solapamiento de contenidos; y comprobar si hay otros aspectos del currículum del Máster que acaban no impartiéndose en ninguna asignatura por la falta de diálogo y trabajo común entre el profesorado. Para ello, el enfoque metodológico adoptado ha sido cuantitativo, aunque el instrumento utilizado en la investigación, un cuestionario, contenga preguntas abiertas analizadas con técnicas de análisis cualitativo. De este modo, la información estadística recogida y expuesta en varias tablas se combina con la reflexión e interpretación de las frases más representativas de las aportaciones y relatos del alumnado. Como indican Bolívar y Domingo (2019), el verdadero potencial de este tipo de investigación basada en las narraciones de sus protagonistas "reside en el hecho de generar conocimiento que ayuda a comprender e interpretar la realidad educativa y constituye una potente herramienta, especialmente pertinente para entrar en el mundo de la identidad, de los significados y del saber práctico" ( $p$. 24). Se trata de dar voz a un elemento que tiene que ser el centro del proceso de enseñanzaaprendizaje, y que muchas veces es silenciado, el alumno.

El estudio se complementa y profundiza mediante la comparación de los resultados obtenidos en el Máster de Formación del Profesorado de la especialidad de Geografía e Historia en las dos universidades en las que se ha realizado la investigación: Universidad de Alicante y Universidad de Valencia.

\subsection{Instrumento}

El instrumento al que se recurre para recoger la información ha sido un cuestionario previamente validado por cuatro docentes de la Universidad de Alicante y de Valencia. En su diseño se han tomado en consideración los criterios propuestos por el ICE de la Universidad de Barcelona (Fernández Núñez, 2007). Para aceptar la estimación del cuestionario se ha tenido en cuenta que con un nivel de confianza 0,95 y un margen de error $7 \%$ se requería recoger 71 cuestionarios, cantidad que ha sido superada al disponer de 73 con lo queda garantizado uno de los requisitos estadísticos mínimos. El tamaño de la muestra proporciona una seguridad estadística, dado que dentro de los límites estimados los resultados obtenidos representan fielmente al universo (Rodríguez Osuna, Ferreras y Núñez, 1991).

El cuestionario está organizado en dos bloques, el primero encaminado a la recogida de los datos sociométricos tales como edad, género, universidad y grado estudiado, y el segundo a la obtención de la información específica sobre la coordinación docente en el Máster. En este análisis cabe advertir que los cuestionarios fueron cumplimentados por los estudiantes en formato virtual al final de la formación en el Máster y no en una fase inicial o intermedia. Además, los encuestados ya habían realizado o estaban finalizando sus prácticas en los centros escolares con lo que podían comparar la formación teórica recibida con las necesidades que generaban su contacto con la realidad del aula.

La primera pregunta analizada del cuestionario es la única que procede del primer bloque y se relaciona con la formación de los participantes, siendo su objetivo obtener información para caracterizar la muestra. La segunda pregunta tiene como intención analizar la coordinación entre las diferentes materias impartidas en la especialidad de Ciencias Sociales del Máster. Esta pregunta es de opción múltiple por lo que se pueden obtener respuestas ubicadas en más de una categoría por cada encuestado. La tercera pregunta es cerrada, marcando los encuestados 
una de las opciones que se les ofrece y está destinada a descubrir si ha habido solapamiento de contenidos con la formación previa al Máster. En la siguiente de las cuestiones planteadas, de naturaleza abierta, el alumnado tenía que concretar, si había existido ese solapamiento, en qué aspectos concretos se había producido. La quinta pregunta tiene como objetivo conocer los contenidos que consideran los encuestados que deberían haber formado parte de sus estudios y se trata también de una pregunta abierta.

El análisis de las preguntas cerradas se ha realizado a través del programa Excel, mientras que en las dos preguntas abiertas se ha utilizado el programa de análisis cualitativo NVivo, categorizándose toda la información recibida del alumnado. Resulta importante señalar -para la comprensión de los resultados mostrados en el apartado 3.1- que para el análisis de la segunda pregunta se han distinguido dos grupos de materias: el de las que integran el Módulo Genérico -de naturaleza psicopedagógica y sociológica- por un lado y el de las relacionadas con la enseñanza de la especialidad de Geografía e Historia por otro. En la Tabla 1 se detallan las materias que los integran. En las materias relacionadas con la especialidad se incluyen las didácticas, las materias de complementos y las de investigación e innovación.

Tabla 1

Materias que integran el Máster en las dos universidades

\begin{tabular}{|c|c|c|c|}
\hline \multicolumn{2}{|l|}{ Universidad de Alicante } & \multicolumn{2}{|l|}{ Universidad de Valencia } \\
\hline Módulo/Materias & Créditos & Módulo/materias & Créditos \\
\hline $\begin{array}{l}\text { Genérico. De naturaleza } \\
\text { psicopedagógica y sociológica }\end{array}$ & 14 & $\begin{array}{l}\text { Genérico. De naturaleza } \\
\text { psicopedagógica y sociológica }\end{array}$ & 16 \\
\hline $\begin{array}{l}\text { Aprendizaje y desarrollo en la } \\
\text { adolescencia }\end{array}$ & 5 & $\begin{array}{l}\text { Aprendizaje y desarrollo de la } \\
\text { personalidad }\end{array}$ & 4 \\
\hline $\begin{array}{l}\text { Diseño y adaptación curricular. } \\
\text { Disfunciones del aprendizaje y del } \\
\text { desarrollo en la adolescencia }\end{array}$ & 3 & Procesos y contextos Educativos & 8 \\
\hline & 3 & Sociedad, familia y educación & 4 \\
\hline $\begin{array}{l}\text { Relacionadas con la enseñanza propia } \\
\text { de Geografía e Historia }\end{array}$ & 30 & $\begin{array}{l}\text { Relacionadas con la enseñanza propia } \\
\text { de Geografía e Historia }\end{array}$ & 28 \\
\hline \multicolumn{4}{|c|}{ Didácticas } \\
\hline $\begin{array}{l}\text { Didáctica de las Ciencias Sociales: } \\
\text { Geografía } \\
\text { Didáctica de las Ciencias Sociales: }\end{array}$ & 7,50 & $\begin{array}{l}\text { Aprendizaje y Enseñanza de la } \\
\text { Geografía y la Historia }\end{array}$ & 16 \\
\hline $\begin{array}{l}\text { Historia, Historia del arte y Educación } \\
\text { para la ciudadanía }\end{array}$ & 7,50 & & \\
\hline
\end{tabular}




\begin{tabular}{lccc}
\hline & \multicolumn{3}{l}{ Complementos } \\
\hline $\begin{array}{l}\text { Geografía en la Enseñanza Secundaria } \\
\text { Historia: de los orígenes a la época }\end{array}$ & 3 & $\begin{array}{l}\text { Complementos para la Formación } \\
\text { disciplinar de Geografía e Historia }\end{array}$ & 6 \\
$\begin{array}{l}\text { moderna } \\
\text { CFD para la enseñanza de la Historia } \\
\text { contemporánea y la Historia del arte } \\
\text { en materias de ESO y Bachillerato }\end{array}$ & 3 & & \\
& 3 & & \\
\hline $\begin{array}{l}\text { Iniciación a la innovación e } \\
\text { investigación educativa en las Ciencias }\end{array}$ & 3 & $\begin{array}{l}\text { Innovación docente e Iniciación a la } \\
\text { investigación educativa especialidad } \\
\text { Sociales: Geografía }\end{array}$ \\
$\begin{array}{l}\text { Innovación e investigación educativa } \\
\text { en la enseñanza Ciencias Sociales: } \\
\text { Historia, Historia del arte y ed. } \\
\text { ciudadanía }\end{array}$ & 3 & \\
\hline
\end{tabular}

Fuente: elaboración propia.

\subsection{Participantes}

La población inicial está integrada por un total de 108 personas matriculadas en el Máster en la especialidad de Ciencias Sociales durante el curso 2020-21, de las cuales han respondido al cuestionario 73 estudiantes. En la Universidad de Alicante de un total de 30 matriculados han respondido 17 , el $56,66 \%$, y en la Universidad de Valencia de un total de 78 matriculados el cuestionario fue implementado por 56 estudiantes lo que corresponde al 71,79\% de los mismos.

Otra característica de la muestra viene definida por la edad de los participantes, que oscila entre los 22 y los 43 años, siendo la media de edad de 26,77 años. Respecto al género, su distribución está muy equilibrada, con un número ligeramente superior de hombres, 50,7\%. La composición de la muestra por el Grado que se ha estudiado se recoge en la Tabla 2, donde se observa cómo, en el conjunto de las dos universidades, está integrada en gran medida por graduados de las especialidades de Historia $(53,42 \%)$ y de Historia del Arte $(26,03 \%)$. La diferencia más significa en la comparación entre las dos universidades corresponde al menor número de Grados que recoge la Universidad de Alicante con solo cuatro Grados, frente a la de Valencia con diez.

Tabla 2

Procedencia del alumnado según Grados cursados y universidad

\begin{tabular}{lcccccc}
\hline \multicolumn{1}{c}{ Grado } & \multicolumn{2}{c}{ U. Alicante } & \multicolumn{2}{c}{ U. Valencia } & \multicolumn{2}{c}{ Total } \\
\cline { 2 - 7 } & $\mathrm{n}$ & $\%$ & $\mathrm{n}$ & $\%$ & $\mathrm{n}$ & $\%$ \\
\hline Geografía & 2 & 11,76 & 3 & 5,36 & 5 & 6,85 \\
Historia & 8 & 47,06 & 31 & 55,36 & 39 & 53,42 \\
Historia del Arte & 4 & 23,53 & 15 & 26,78 & 19 & 26,03 \\
Historia y Geografía & 0 & 0,00 & 1 & 1,79 & 1 & 1,37 \\
Humanidades & 3 & 17,65 & 0 & 0,00 & 3 & 4,11 \\
Otros & 0 & 0,00 & 6 & 10,71 & 6 & 8,22 \\
Participantes & 17 & 100,00 & 56 & 100,00 & 73 & 100,00 \\
\hline
\end{tabular}

Fuente: elaboración propia. 


\section{Resultados}

Siguiendo el objetivo inicial de analizar la percepción del alumnado sobre la coordinación existente dentro del Máster de Profesorado de Enseñanza Secundaria en la especialidad de Ciencias Sociales -Geografía e Historia- se han obtenido los siguientes resultados que se presentan organizados en dos subapartados. En el primero descubriremos las relaciones dentro de las materias y entre las materias y en el segundo se prestará atención a las presencias, ausencias y repeticiones en los contenidos.

\subsection{La coordinación entre las materias}

Del análisis de la Tabla 1, que hacía referencia a las materias que integran el Máster en las dos universidades analizadas en el estudio, se infiere que en el Módulo Genérico en la Universidad de Alicante hay más materias -cuatro frente a tres-y menos créditos-catorce frente a dieciséisque en la Universidad de Valencia, y en las de la especialidad, relacionadas con la enseñanza propia de Geografía e Historia, también el número de materias es bastante mayor en la primera, a pesar de que el número de créditos es ligeramente superior. Esta estructura organizativa del Máster hace suponer que inicialmente en la Universidad de Alicante la coordinación tendrá más dificultades que en la de Valencia debido a ese mayor número de asignaturas.

No obstante, el análisis que se presenta a continuación acerca de los grupos de materias en los que ha existido una coordinación menor y cuyos datos se recogen en la Tabla 3 -cada uno de los participantes podía elegir más de una opción-viene a cuestionar esta primera impresión. Dentro del Módulo Genérico, cabe destacar que la coordinación en general ha sido mayor, pues solo el $28,77 \%$ de los estudiantes considera que ha faltado coordinación. La información disponible permite afirmar que un mayor número de asignaturas no ha implicado en la Universidad de Alicante una mayor falta de coordinación $(17,65 \%)$ que en la de Universidad de Valencia $(32,14 \%)$, sino al contrario.

Sin embargo, las materias de la especialidad de Geografía e Historia son las que tienen menos coordinación (35,62\%). Estas diferencias en la falta de coordinación varían según el contexto de cada universidad. Mientras en la Universidad de Valencia la percepción de falta de coordinación es la misma para el bloque de naturaleza psicopedagógica-sociológica y el de especialidad $(32,14 \%)$, en la Universidad de Alicante es significativa la diferencia entre ambos bloques, ya que el $47,06 \%$ de los participantes percibe una mayor falta de coordinación en las materias de la especialidad frente a las del módulo genérico $(17,65 \%)$.

Si el análisis se traslada a la coordinación entre las materias del Módulo Genérico y las de especialidad el problema se agrava, pues más de la mitad de los universitarios $(50,68 \%)$ consideran que la coordinación entre ambas es escasa. Una vez más los datos permiten inferir que este problema depende de factores contextuales, pues es significativo que en la Universidad de Alicante la falta de coordinación la señala el 35,29\% de los jóvenes y en la Universidad de Valencia el $55,36 \%$.

En el análisis cualitativo recogemos varios comentarios que van en la misma línea, porque posiblemente alberguen una de las principales razones que explican la deficiencia en la coordinación "las asignaturas son más bien compartimentos estancos" (G27). Además, si la asignatura esta compartida por varios docentes el problema es aún mayor según se deduce de varios testimonios: "Falta de coordinación entre los profesores de la asignatura" (H11) o menor coordinación en materias en "Las que tenían más de un profesor" (H24). 
Tabla 3

Grupos de materias en los que ha habido menos coordinación

\begin{tabular}{lcccccc}
\hline $\begin{array}{l}\text { Materias entre las que ha faltado más } \\
\text { coordinación }\end{array}$ & \multicolumn{2}{c}{ U. Alicante } & \multicolumn{2}{c}{ U. Valencia } & \multicolumn{2}{c}{ Total } \\
\cline { 2 - 7 } & $\mathrm{n}$ & $\%$ & $\mathrm{n}$ & $\%$ & $\mathrm{n}$ & $\%$ \\
\hline $\begin{array}{l}\text { En las de naturaleza psicopedagógica y } \\
\text { sociológica }\end{array}$ & 3 & 17,65 & 18 & 32,14 & 21 & 28,77 \\
$\begin{array}{l}\text { En las relacionadas con la enseñanza } \\
\text { propia de la Geografía e Historia }\end{array}$ & 8 & 47,06 & 18 & 32,14 & 26 & 35,62 \\
$\begin{array}{l}\text { Entre las de naturaleza psicopedagógica- } \\
\text { sociológica y las relacionadas con }\end{array}$ & 6 & 35,29 & 31 & 55,36 & 37 & 50,68 \\
$\begin{array}{l}\text { Geografía e Historia } \\
\text { Participantes }\end{array}$ & 17 & 100,00 & 56 & 100,00 & 73 & 100,00 \\
\hline
\end{tabular}

Fuente: elaboración propia.

\subsection{Contenidos repetidos versus ausentes}

Si el análisis cualitativo y cuantitativo ha posibilitado evidenciar el grave problema de coordinación en el Máster de Formación del Profesorado, cabe avanzar igualmente en otra cuestión de interés: profundizar en los contenidos que se enseñan. Para este objetivo se analiza primero las repeticiones de contenidos y en segundo lugar las ausencias o carencias que señalan los participantes y que consideran esenciales para cubrir sus necesidades formativas.

En la Tabla 4 se presenta los resultados sobre la repetición de contenidos entre la formación recibida en el Grado y la que han tenido en el Máster. La información procede de una pregunta cerrada en la que se distinguen cinco niveles que van desde un solapamiento total, opción de "Todos", a la contraria "Ninguno". Se observa que el $84,93 \%$ de los jóvenes de ambas universidades ha indicado que no hay solapamiento o apenas ocurre este hecho, por lo que se puede inferir inicialmente que el problema de repetición de contenidos entre ambos estudios, Grado-Máster, no es grave. Sin embargo, sí se han detectado diferencias significativas entre universidades, ya que en la Universidad de Alicante el 53,94\% de los encuestados considera que sí se produce reiteración, opción de "Bastantes" y "Muchos", mientras que en la de Valencia el 96,43\% indica que apenas hay repetición de contenidos, opción "Ninguno" y "Poco".

Tabla 4

Contenidos tratados en el Grado de procedencia y repetidos en el Máster

\begin{tabular}{lcccccc}
\hline Contenidos & \multicolumn{2}{c}{ U. Alicante } & \multicolumn{2}{c}{ U. Valencia } & \multicolumn{2}{c}{ Total } \\
\cline { 2 - 6 } repetidos & $\mathrm{n}$ & $\%$ & $\mathrm{n}$ & $\%$ & $\mathrm{n}$ & $\%$ \\
\hline Ninguno & 1 & 5,88 & 18 & 32,14 & 19 & 26,03 \\
Poco & 7 & 41,18 & 36 & 64,29 & 43 & 58,9 \\
Bastantes & 8 & 47,06 & 0 & 0,00 & 8 & 10,96 \\
Muchos & 1 & 5,88 & 2 & 3,57 & 3 & 4,11 \\
Todos & 0 & 0,00 & 0 & 0,00 & 0 & 0,00 \\
Participantes & 17 & 100,00 & 56 & 100,00 & 73 & 100,00 \\
\hline
\end{tabular}

Fuente: elaboración propia. 
Obviamente, considerando que en los Grados de procedencia no hay asignaturas de psicopedagogía y previendo que hubiera una posible respuesta de solapamientos desde las ciencias de referencia o especialidad se incluyó otra pregunta, de naturaleza abierta, que permitiera aflorar y detallar dónde se podría producir esta repetición de contenidos.

Los resultados de este análisis se presentan en la Tabla 5 -cada uno de los participantes podía elegir una o varias opciones, incluso ninguna-. Cabe advertir que como la procedencia de la mayoría del alumnado del Máster es de Historia, resulta comprensible que se alcancen en este Grado el mayor porcentaje de repeticiones $(41,1 \%)$, siendo elevados los porcentajes que se detectan en ambas universidades analizadas. Sin embargo, la repetición de contenidos donde consigue el valor más elevado es en el Grado de Geografía y en Alicante $(52,94 \%)$ a diferencia de Valencia con el $17,86 \%$.

Tabla 5

Contenidos tratados en el Grado de procedencia y repetidos en el Máster, concretados por ciencias de referencia

\begin{tabular}{lcccccc}
\hline Grado de procedencia & \multicolumn{2}{c}{ U. Alicante } & \multicolumn{3}{c}{ U. Valencia } & \multicolumn{2}{c}{ Total } \\
\cline { 2 - 7 } & $\mathrm{n}$ & $\%$ & $\mathrm{n}$ & $\%$ & $\mathrm{n}$ & $\%$ \\
\hline Historia & 8 & 47,06 & 22 & 39,29 & 30 & 41,1 \\
Geografía & 9 & 52,94 & 10 & 17,86 & 19 & 26,03 \\
Arte & 1 & 5,88 & 4 & 7,14 & 5 & 6,85 \\
Género & 0 & 0,00 & 2 & 3,57 & 2 & 2,74 \\
Participantes & 17 & 100,00 & 56 & 100,00 & 73 & 100,00 \\
\hline
\end{tabular}

Fuente: elaboración propia.

Por tanto, con esta información se infiere en primer lugar que sí se produce repetición de contenidos entre los estudios de Grado y de Máster, particularmente en el módulo de especialidad, e igualmente se puede afirmar que esta repetición también depende de la organización de cada Universidad.

Como indica García-Rubio (2021, p. 60) "La materia de Complementos absorbe el 34 \% de los créditos del Módulo de Formación Específica; más de un tercio de dichos créditos son impartidos por departamentos ajenos a las didácticas específicas". Por tanto, la procedencia del profesorado puede ayudar a entender que se repitan los contenidos del Máster y los del Grado.

Por ello, la investigación se ha encaminado a descubrir y detallar los contenidos que los encuestados consideraban necesarios y que se han impartido de forma insuficiente o no han sido incorporados a su formación docente. En la Tabla 6 se visualiza el resultado de la pregunta de naturaleza abierta incluida en el cuestionario sobre esta problemática. La disposición de contenidos en esta tabla viene determinada por el valor del porcentaje obtenido en el conjunto de las dos universidades, y distribuidos de mayor a menor. 
Tabla 6

Contenidos no impartidos en ninguna asignatura y que se deberían de haber dado en el Máster

\begin{tabular}{|c|c|c|c|c|c|c|}
\hline \multirow{2}{*}{$\begin{array}{l}\text { Contenidos que se deberían de haber } \\
\text { dado }\end{array}$} & \multicolumn{2}{|c|}{ U. Alicante } & \multicolumn{2}{|c|}{ U. Valencia } & \multicolumn{2}{|c|}{ Total } \\
\hline & $\mathrm{n}$ & $\%$ & $\mathrm{n}$ & $\%$ & $\mathrm{n}$ & $\%$ \\
\hline $\begin{array}{l}\text { Atención a la diversidad, inclusión y } \\
\text { necesidades educativas especiales }\end{array}$ & 0 & 0,00 & 25 & 44,64 & 25 & 34,25 \\
\hline Más relacionados con la práctica & 3 & 17,65 & 7 & 12,50 & 10 & 13,70 \\
\hline Programación de unidades didácticas & 0 & 0,00 & 10 & 17,86 & 10 & 13,70 \\
\hline $\begin{array}{l}\text { Situaciones reales de aula: acoso escolar, } \\
\text { maltrato familiar }\end{array}$ & 3 & 17,65 & 7 & 12,50 & 10 & 13,70 \\
\hline Salidas profesionales, oposición & 0 & 0,00 & 9 & 16,07 & 9 & 12,33 \\
\hline $\begin{array}{l}\text { Más psicopedagogía y no tanto de } \\
\text { especialidad }\end{array}$ & 4 & 23,53 & 4 & 7,14 & 8 & 10,96 \\
\hline Hemos dado lo esperado & 2 & 11,76 & 5 & 8,93 & 7 & 9,59 \\
\hline TIC & 1 & 5,88 & 6 & 10,71 & 7 & 9,59 \\
\hline $\begin{array}{l}\text { Más contenidos de las asignaturas de la } \\
\text { especialidad }\end{array}$ & 4 & 23,53 & 1 & 1,79 & 5 & 6,85 \\
\hline Comunicación y expresividad & 3 & 17,65 & 1 & 1,79 & 4 & 5,48 \\
\hline Metodologías innovadoras & 0 & 0,00 & 4 & 7,14 & 4 & 5,48 \\
\hline Relación con el alumnado & 2 & 11,76 & 2 & 3,57 & 4 & 5,48 \\
\hline Evaluación & 0 & 0,00 & 3 & 5,36 & 3 & 4,11 \\
\hline Control del aula & 0 & 0,00 & 2 & 3,57 & 2 & 2,74 \\
\hline Género & 0 & 0,00 & 2 & 3,57 & 2 & 2,74 \\
\hline Tareas burocráticas docente & 0 & 0,00 & 2 & 3,57 & 2 & 2,74 \\
\hline Legislación & 1 & 5,88 & 0 & 0,00 & 1 & 1,37 \\
\hline Motivación alumnado & 1 & 5,88 & 0 & 0,00 & 1 & 1,37 \\
\hline Tutoría & 0 & 0,00 & 1 & 1,79 & 1 & 1,37 \\
\hline Participantes & 17 & 100,00 & 56 & 100,00 & 73 & 100,00 \\
\hline
\end{tabular}

Fuente: elaboración propia.

La disposición de las categorías en la tabla, ordenadas por frecuencias, no oculta que la mayoría de los contenidos que echa en falta el alumnado corresponden al Módulo Genérico, mientras que paradójicamente las repeticiones y solapamientos se producían con mayor frecuencia en el bloque de materias de especialidad. No obstante, el análisis ofrece aspectos de detalle significativos.

En primer lugar, hay que destacar que sólo el 9,59\% indica que han recibido la formación que esperaban y esta opinión es una de las que muestra menos diferencia entre ambas universidades. Sin duda este porcentaje invita a una profunda reflexión sobre la formación docente del Máster.

La categoría con mayor porcentaje de ausencia es "Atención a la diversidad, inclusión y necesidades educativas especiales" con el $44,64 \%$ en Valencia. Sin embargo, en Alicante, ni siquiera se cita esta ausencia. Se manifiesta más las diferencias entre universidades que las intrínsecas a las materias. El análisis cualitativo de esta categoría permite conocer mejor esta circunstancia. En la Universidad de Valencia, uno de los estudiantes indica que "se han mencionado muchísimo pero no hemos visto ejemplos de actividades multinivel o algún otro tipo de implementación para la inclusión de estos aspectos en las dinámicas de aula" (K48) y 
otro es muy crítico al respecto señalando que "la formación en atención a la diversidad es absolutamente deficiente" (K38).

Las diferencias en la categoría denominada "Más relacionados con la práctica" son bastante más reducidas entre ambas universidades, pero en conjunto ocupan una posición relevante, lo que resulta preocupante por la orientación profesionalizante del Máster. Con el mismo porcentaje, $13,70 \%$, se encuentra otra categoría relacionada "Situaciones reales de aula: acoso escolar, maltrato familiar". Igualmente, las diferencias en este caso entre las dos universidades no son de las más significativas, por lo que se puede inferir que son necesidades formativas generalizadas.

El análisis cualitativo de la información permite comprender mejor esta cuestión. Según uno de los estudiantes se tenía que haber impartido "algún contenido relacionado a cómo dar las clases como docentes, o para enfrentarnos a nuestro período de prácticas en el centro educativo. Ya que llegamos a las prácticas sin saber cómo enfrentarnos a la tarea de enseñar" (K2) y un segundo de ellos señala que "me ha faltado más contenido práctico dentro de las propias materias, dejando al margen el Prácticum. Me ha parecido un máster muy centrado en la investigación y en la teoría, pero poco centrado en la práctica docente" (K67). Esa necesidad de mayor practicidad también se constata en el tratamiento de más situaciones reales del aula como bullying, maltrato familiar, etc., siendo los porcentajes idénticos a la categoría anterior. Uno de los participantes manifiesta que "se podría dedicar más tiempo a la praxis del docente ante problemas de la clase (situaciones violentas, cómo actuar ante cualquier imprevisto)" (K51).

La formación sobre programaciones didácticas también es una de las carencias más presentes en las respuestas, aunque el problema se centra exclusivamente en la Universidad de Valencia (17,86\%). El análisis cualitativo permite avanzar más en esta problemática, que enlaza con la patología del didactismo consistente en suponer que al alumnado ya se lo han explicado. Según uno de los encuestados la necesidad de "Explicar pormenorizadamente cómo se realiza una Unidad didáctica. ¿Qué es una Unidad Didáctica? En ninguna asignatura se tomaron la molestia de explicarlo" (K24).

Otra carencia manifestada es de otra naturaleza y está relacionada con las salidas profesionales. También existe disparidad entre las dos universidades. En la Universidad de Valencia hay una fuerte percepción de que se han de tratar las salidas profesionales docentes y más en concreto la oposición (16,07\%). Los estudiantes de la Universidad de Alicante no reclaman este contenido. Uno de los estudiantes universitarios de Valencia señala que se debería "profundizar en cuestiones más administrativas que de verdad nos sirvan de cara a unas oposiciones o de cara a trabajar en un centro educativo" (K31).

Asimismo, cabría señalar la necesidad de incorporar TIC según el 9,59\% y aunque el porcentaje ya no es de los más elevados, no deja de preocupar en una sociedad que avanza en la digitalización de todas las actividades, y en un contexto de pandemia donde el profesorado ha realizado un gran esfuerzo para estar a la altura de las circunstancias.

Para concluir el análisis cabe manifestar que las respuestas de los jóvenes deben ser consideradas de forma orientativa, pues ellos mismos pueden llegar a posiciones contradictorias como ocurre en la Universidad de Alicante entre quienes consideran que debería haber "Más psicopedagogía y no tanto de especialidad" $23,53 \%$ y por el contrario quienes opinan que debería existir más "Asignaturas de la especialidad y más contenidos" con igualmente el 23,53\%. El análisis cualitativo una vez más viene a complementar el cuantitativo. De este modo en una respuesta se indica "que debería de haber más asignaturas con contenido psicológico o psicopedagógico y no tantas de materias como Historia o Geografía que ya hemos cursado en la carrera y son meras repeticiones de contenidos" (K3), mientras que en otra se manifiesta que 
"he echado en falta una mayor profundización en aspectos metodológicos concretos sobre la disciplina, tanto en historia como en geografía" (K42).

Para resolver la contradicción en el relato sobre qué tipo de materias resultaría imprescindible incorporar al currículum, según el alumnado, se ha elaborado la Tabla 7, donde se han agrupado las respuestas dadas y recogidas en la tabla anterior en tres categorías, tratando de relacionarlas con las materias psicopedagógicas y sociológicas, o bien con las de la enseñanza de Geografía e Historia. A partir de esta categorización se puede comprobar que los contenidos que echan en falta están mucho más relacionados con la primera de las categorías citadas $(54,78 \%)$ que con la didáctica propia de su especialidad (22,61\%). Los estudiantes y futuros profesores de Enseñanza Secundaria consideran que sería necesario impartir, o hacerlo en mayor profundidad, contenidos relacionados con la atención a la diversidad, inclusión y necesidades educativas especiales, así como abordar situaciones reales de aula, comunicación del profesorado, trato con los alumnos, gestión y clima de aula, etc. También en relación con la especialidad de Geografía e Historia destacan cuestiones como la realización de programaciones de unidades didácticas, el uso de las TIC y de metodologías innovadoras. Carro Sancristóbal (2000) ya detectó en su investigación las circunstancias analizadas señalando que el alumnado del CAP en aquellos años consideraba que "no se les ha enseñado la suficiente pedagogía para ser un buen profesional..." (p. 17) y entre las soluciones proponía la que en esta investigación se presenta como una cuestión pendiente: "Frente a una situación de independencia, en el que cada profesional actúa de forma aislada e individualista, la propuesta de cambio se sitúa en unos términos de interdependencia, comunicación y colaboración. Los profesionales no están solos y cada actuación individual no puede estar fuera de las coordenadas de grupo" (p. 26).

Tabla 7

Contenidos que se deberían haber dado en el Máster, ordenados por grupos de materias

\begin{tabular}{|c|c|c|c|c|c|c|}
\hline \multirow[t]{2}{*}{ Contenidos que se deberían haber dado } & \multicolumn{2}{|c|}{ U. Alicante } & \multicolumn{2}{|c|}{ U. Valencia } & \multicolumn{2}{|c|}{ Total } \\
\hline & $\mathrm{n}$ & $\%$ & $\mathrm{n}$ & $\%$ & $\mathrm{n}$ & $\%$ \\
\hline $\begin{array}{l}\text { En las de naturaleza psicopedagógica y } \\
\text { sociológica }\end{array}$ & 14 & 58,34 & 49 & 53,84 & 63 & 54,78 \\
\hline $\begin{array}{l}\text { En las relacionadas con la enseñanza } \\
\text { propia de la Geografía e Historia }\end{array}$ & 5 & 20,83 & 21 & 23,08 & 26 & 22,61 \\
\hline $\begin{array}{l}\text { Otras respuestas no clasificables en las } \\
\text { dos agrupaciones de materias }\end{array}$ & 5 & 20,83 & 21 & 23,08 & 26 & 22,61 \\
\hline Total de contenidos señalados & 24 & 100,00 & 91 & 100,00 & 115 & 100,00 \\
\hline
\end{tabular}

Fuente: elaboración propia.

\section{Discusión}

A partir de estos resultados alcanzados se abre una cuestión para el debate relacionado con el modelo de coordinación, las capacidades y limitaciones asignadas y las funciones desempeñadas en la coordinación formal de la especialidad. Generalmente la persona designada tiene encomendada una función más de informar sobre cuestiones genéricas y de recopilar datos estadísticos, que de armonizar las materias. De la investigación se infiere la necesidad ineludible de replantearse el modelo de coordinación existente. Los datos recogidos, especialmente en la Tabla 3, sugieren la conveniencia de establecer una propuesta general de intervención en la coordinación, pero con un seguimiento particularizado, teniendo en cuenta entre otros criterios la realidad de cada universidad y de las materias. Así, por ejemplo, Gutiérrez Rodríguez, Guillén y Jaime Pastor (2012), respecto a la especialidad de matemáticas en el Máster de Profesorado 
de Educación Secundaria en la Universidad de Valencia, también sugieren importantes aspectos a modificar en la coordinación entre las asignaturas.

Inicialmente, por el alcance de los datos recogidos en la presente investigación, se hace imprescindible actuar en la relación entre materias de un mismo módulo, y en segundo lugar dentro de éstos, particularmente en las de especialidad, pues los resultados dependen también del número de docentes que imparten la asignatura, como pone de manifiesto el análisis cualitativo. Una de las peculiaridades bastante habituales en este Máster, es la de que una misma materia sea dividida entre varios profesores, incluso a veces como se indica en algún testimonio en un número desproporcionadamente alto -seis profesores- (García-Rubio, 2021). En este caso, la primera labor en relación con la coordinación sería la de establecer una guía didáctica consensuada por todo el profesorado participante (Fuentes-Guerra et al., 2012), de manera que los contenidos y actividades estuviesen perfectamente armonizados. Sin embargo, además de la coordinación entre el profesorado que comparte una asignatura, no es menos importante la que se debe establecer entre los docentes de las distintas materias del Máster, unas más genéricas y relacionadas con aspectos psicopedagógicos, y otras más vinculadas con las diferentes especialidades que cursa el alumnado (Hernández Amorós y Carrasco Embuena, 2012).

La preocupación inicial sobre qué modelo de coordinación resulta más pertinente queda superado por la realidad del modelo vigente en dos universidades españolas (Alicante, Valencia), donde se ha detectado un grave problema de coordinación entre el módulo general (psicología, sociología) y el de didácticas específicas, y además entre estas dos categorías. Profundizando en esta cuestión, se ha detectado el problema de las repeticiones de contenidos, que se hace más patente en la formación didáctica relacionada con la especialidad, y al mismo tiempo la ausencia de contenidos que son considerados necesarios en su formación por el alumnado del Máster.

Tanto el análisis cuantitativo como el cualitativo realizado, vienen a coincidir en el interés y demanda de contenidos que se consideran que han estado poco presentes y que desde la perspectiva del estudiante serán esenciales en el ejercicio de su labor docente. El análisis realizado permite afirmar que existe la necesidad de revisar los contenidos a impartir en la formación inicial del profesorado de Enseñanza Secundaria en la línea señalada por ImbernónMuñoz (2021, p. 33), quien indica que el futuro profesorado "debe asumir más un papel práctico reflexivo en una cultura de la colaboración". Resulta imposible adquirir todos los aprendizajes en el Máster, pero algunos se deberían de considerar como básicos, de difícil olvido en el currículum de formación del futuro profesor de Enseñanza Secundaria. Una buena formación inicial es primordial para que el ejercicio inicial de la profesión no sea excesivamente complicado (Vaillant, 2008), pero también es donde se sientan las bases de su futuro desarrollo profesional (Day, 2005; Imbernón-Muñoz, 2019).

Asimismo, las muy distintas denominaciones de las asignaturas del Máster en la especialidad de Geografía e Historia revelan "la incertidumbre en la definición del objeto de estudio" (Souto González, 2017, p. 21). Un problema específico de esta especialidad del Máster es que su alumnado suele ser graduado en Historia o en Geografía o en Historia del Arte, es decir no han cursado un Grado que les posibilite el dominio de unos conocimientos adecuados en las tres disciplinas. Por lo tanto, esta será una tarea añadida a su formación como docente en la especialidad del Máster (Rivero Gracia y Souto González, 2019), además de la de posibilitar una preparación amplia que les posibilite el éxito en el ejercicio de la profesión en su fase inicial.

Surge en este momento una duda que se transforma en una cuestión para la discusión ¿Se imparte realmente una didáctica de especialidad o los docentes proceden simplemente a repetir contenidos de la materia, es decir, de Geografía e Historia y no de didáctica de la Geografía y de la Historia? Sin duda esta cuestión queda abierta, pero los datos recogidos particularmente en 
la Tabla 5 nos indican que la didáctica en las materias específicas en el Máster se limita en muchos casos a repetir contenidos de los Grados de procedencia. Por tanto, si se decide intervenir en el modelo de formación docente, otra cuestión procedería plantearse son los contenidos que se enseñan y la necesidad de una elección de los formadores más orientada hacia la didáctica.

Por otra parte, para la mejora de futuras investigaciones que se pretenden realizar con el propósito de ampliar el conocimiento acerca de la coordinación docente del Máster de Formación del Profesorado en la especialidad de Geografía e Historia, sería conveniente recoger la visión de sus docentes, tanto de forma individual como a través de grupos de discusión. Su perspectiva complementaría la que ya se ha obtenido del alumnado.

\section{Conclusiones}

Una primera conclusión recogida es que el problema de coordinación no depende esencialmente del número de asignaturas, sino más bien de cómo se organizan los estudios, y esta cuestión se evidencia en las diferencias entre universidades. Las dificultades en la coordinación resultan más patentes en las asignaturas específicas que en las materias relacionadas con la psicopedagogía y sociología. Una de las razones que manifiestan los jóvenes participantes en el cuestionario es que las asignaturas de especialidad, algunas de ellas citadas reiteradamente, son impartidas por varios docentes, lo que dificulta su coordinación, a pesar de que en muchas ocasiones procedan del mismo departamento.

Una segunda cuestión ha sido la concreción de los problemas de coordinación en una doble dirección. Por un lado, se ha confirmado la repetición de muchos de los contenidos que configuran la formación de los futuros profesores de Enseñanza Secundaria de Geografía e Historia, lo que lleva a la falta de aprovechamiento del escaso tiempo de formación del Máster y también a la falta de interés del alumnado en esos contenidos reiterados. Por otro lado, la ausencia de contenidos que los estudiantes consideran oportunos nos lleva irremediablemente a la necesidad de establecer una coordinación docente en el Máster de un modo completamente diferente a la que se realiza en la actualidad, a todas luces insuficiente, y que requiere del compromiso del profesorado en su labor esencial de formación.

Se requiere asimismo y, sobre todo, del apoyo y la determinación del conjunto de la universidad española en la consecución de una formación inicial de extraordinaria calidad, donde la coordinación docente sea una práctica profesional interiorizada y plenamente asumida.

\section{Referencias}

Ávalos, B., Cavada, P., Pardo, M. \& Sotomayor, C. (2010). La profesión docente: temas y discusiones en la literatura internacional. Estudios Pedagógicos, 36(1), 235-263.

Beane, J. A. (2005). La integración del currículum. Ediciones Morata.

Bernstein, B. (1973). On the classification and framing of educational knowledge. En R. Brown (Ed). Knowledge, education, and cultural change. Papers in the sociology of education (pp. 360-392). Routledge.

Bolarín Martínez, M. J., Moreno Yus, M. Á. y Porto Currás, M. (2013). Coordinación docente e interdisciplinariedad: análisis de su contribución a la adquisición de competencias docentes y discentes. REDU - Revista de Docencia Universitaria, 11(2), 443-462. https://doi.org/10.4995/redu.2013.5584 
Bolarín Martínez, M. J., y Moreno Yus, M. Á. (2015). La coordinación docente en la universidad: retos y problemas a partir de Bolonia. Profesorado. Revista de Currículum y Formación de Profesorado, 19(2), 319-332.

Bolívar, A. y Domingo, J. (2019). La investigación (auto) biográfica en educación. Octaedro.

Carro Sancristóbal, L. (2000). La formación del profesorado en investigación educativa: una visión crítica. Revista Interuniversitaria de Formación del Profesorado, 39, 15-32.

Crespo Castellanos, J. M., Sebastiá Alcaraz, R. y Souto González, X. M. (2021). La enseñanza de la Geografía en el Máster de Formación del Profesorado. En C. J. Gómez Carrasco, X. M. Souto González y P. Miralles Martínez (Eds.). Enseñanza de las Ciencias Sociales para una ciudadanía democrática. Estudios en homenaje al profesor Ramón López Facal (pp. 5669). Octaedro.

Day, C. (2005). Formar docentes. Cómo, cuándo y en qué condiciones aprende el profesorado. Narcea.

Escudero Muñoz, J. M., Campillo Díaz, M., y Sáez Carreras, J. (2019). El Máster de Formación Inicial del Profesorado de Educación Secundaria: revisión, balances y propuestas de mejora. Profesorado, Revista de Currículum y Formación del Profesorado, 23(3), 165-188. http://dx.doi.org/10.30827/profesorado.v23i3.9409

Fernández Núñez, L. I. (2007). ¿Cómo se elabora un cuestionario? Butlletí LaRecerca, ficha 8, ICE: Universitat de Barcelona. https://www.ub.edu/idp/web/sites/default/files/fitxes/ficha8cast.pdf

Fuentes-Guerra, M., García, M. M., Llorent, V. J. y Olivares, M. A. (2012). La coordinación docente universitaria desde la percepción del alumnado. Revista de Docencia Universitaria, 10(2), 395-409.

García Cid, Y. (2013). Una experiencia de coordinación docente en la enseñanza universitaria. Anales de ASEPUMA, 21, 1-11.

García Martín, A. (2015). Coordinación docente horizontal y vertical. CRAl. Universidad Politécnica de Cartagena.

García-Martínez, I. (2019). Coordinación pedagógica y liderazgo distribuido en los institutos de secundaria [Tesis doctoral]. Universidad de Granada. https://digibug.ugr.es/handle/10481/55983

García-Rubio, J. (2021). Cambios para la mejora del máster de formación del profesorado de secundaria en España. En G. Gómez García, C. Rodríguez Jiménez, N. Campos Soto y M. Ramos Navas (Coords.). Nuevos escenarios educativos. Hacia el horizonte 2030 (pp. 550560). Dykinson.

González Sala, F., Bisquert, M., Haba-Osca, J. y Osca-Lluch, M. J. (2020). Formación del profesorado de Secundaria en España: Un estudio a través de los Másteres Oficiales en Educación Secundaria en universidades públicas. Revista Interuniversitaria de Formación del Profesorado, 34(2), 205-224.

Gutiérrez Rodríguez, A., Guillén, G. y Jaime Pastor, A. (2012). Coordinación entre las asignaturas de matemáticas en el máster de profesorado de educación secundaria, Uno, 59, 74-81.

Hernández Amorós, M. J. y Carrasco Embuena, V. (2012). Percepciones de los estudiantes del Máster en Formación del Profesorado de Educación Secundaria: fortalezas y debilidades del nuevo modelo formativo. Enseñanza \& Teaching, 30(2), 127-152. 
Imbernón-Muñoz, F. (2019). La formación del profesorado de Educación Secundaria: la eterna pesadilla. Profesorado. Revista de Currículum y Formación de Profesorado, 23(3), 151-163. https://doi.org/10.30827/profesorado.v23i3.9302

Imbernón-Muñoz, F. (2021). Realidad y retos de la formación inicial permanente del profesorado de Educación Secundaria. En C. J. Gómez Carrasco, X. M. Souto González y P. Miralles Martínez (Eds.). Enseñanza de las ciencias sociales para una ciudadanía democrática (pp. 23-35). Octaedro.

Jarauta Borrasca, B. y Medina Moya, J. L. (2012). Saberes docentes y enseñanza universitaria. Estudios sobre Educación, 22, 179-198.

Lavié Martínez, J. M. (2004). Individualismo y privacidad en la cultura docente: revisando los argumentos. Revista Española de Pedagogía, 229, 439-453.

López Esteban, C. (2016). Propuesta de coordinación horizontal y vertical en el Máster en Profesor de Secundaria, Memoria Final. Repositorio Gredos. https://gredos.usal.es/bitstream/handle/10366/131617/MID_15_191.pdf?sequence=1\& isAllowed=y

Orden $\mathrm{ECl} / 3858 / 2007$, de 27 de diciembre, por la que se establecen los requisitos para la verificación de los títulos universitarios oficiales que habiliten para el ejercicio de las profesiones de Profesor de Educación Secundaria Obligatoria y Bachillerato, Formación Profesional y Enseñanzas de Idiomas. (BOE de 29 de diciembre de 2007).

Paricio Royo, J. (2012). Diez principios para un sistema de gestión de la calidad concebido específicamente para la coordinación y la mejora interna de las titulaciones universitarias. REDU - Revista de Docencia Universitaria, 10(3), 49-69. https://doi.org/10.4995/redu.2012.6014

Perrenoud, P. (2012). Cuando la escuela pretende preparar para la vida. ¿Desarrollar competencias o enseñar otros saberes? Grao.

Real Decreto 1393/2007, de 29 de octubre, por el que se establece la ordenación de las enseñanzas universitarias oficiales. (BOE de 30 de octubre de 2007).

Rivero Gracia, P. y Souto González, X. M. (2019). El máster de profesorado de Educación Secundaria: una visión desde la especialidad de Geografía e Historia. Iber: Didáctica de las Ciencias Sociales. Geografía e Historia, 97, 41-50.

Rodríguez Osuna, J., Ferreras, M. L. y Núñez, A. (1991). Inferencia estadística, niveles de precisión y diseño muestral. Reis, 54, 139-162.

Sánchez Tarazaga, L. (2019). Situación actual del MESOB en España: visión global de sus planes de estudios. En J. Manso (Coord.). La formación inicial del profesorado en España: Análisis de los planes de estudios tras una década desde su implementación (pp. 83-107). Ministerio de Educación.

Sarramona, J. (2007). Las competencias profesionales del profesorado de secundaria. Estudios sobre Educación, 12, 31-40.

Souto González, X. M. (2017). La formación del profesorado de Geografía e Historia. Balance y perspectivas desde el Máster de formación del profesorado de Educación Secundaria. En P. Miralles Martínez, C. J. Gómez Carrasco y R. A. Rodríguez Pérez. La enseñanza de la historia en el siglo XXI. Desarrollo y evaluación de competencias históricas para una ciudadanía democrática (pp. 13-34). Universidad de Murcia. 
Torrego Ejido, L. y Ruiz Esteban, C. (2011). La coordinación docente en la implantación de los títulos de Grado. Revista Electrónica Interuniversitaria de Formación del Profesorado, 14(4), 31-40.

Vaillant, D. (2008). La identidad docente. La importancia del profesorado. Revista Investigaciones en Educación, 8(1), 15-39.

Vaillant, D. y Marcelo, C. (2015). El ABC y D de la formación docente. Narcea.

\section{Agradecimientos}

La investigación presentada está encuadrada en el Proyecto de Título: Las representaciones sociales de los contenidos escolares en el desarrollo de las competencias docentes, Proyectos I+D de Generación del Conocimiento y Fortalecimiento Científico y Tecnológico del Sistema I+D+I (BOE 28-08-2018). 